\title{
Introduction: Special Issue on Atom Probe Tomography
}

In February 2006, in conjunction with the 19th Australian Conference on Microscopy and Microanalysis held in Sydney, the 2nd Australian Workshop on Atom Probe Tomography was convened by S.P. Ringer, M.K. Miller, D.A. Saxey, and R. Zheng at the Australian Key Centre for Microscopy and Microanalysis at The University of Sydney. The topics covered at that workshop included specimen preparation; data acquisition and data analysis methods for atom probe tomography; applications to spinodal alloys, phase transformations, light metals, atomic clustering, and detection methods, as well as future directions of the science and technology of atom probe tomography. The presentations and discussions that took place at this workshop, which was attended by more than 30 people, provided the inspiration for this special issue of Microscopy and Microanalysis.

As pointed out in the introductory overview paper by Cerezo et al., recent advances in high-resolution microscopy across the areas of atom probe tomography, aberrationcorrected transmission electron microscopy, and electron tomography, to list but a few, are very significant. Particularly in the area of atom probe, there has been a related stream of innovation in specimen preparation based largely around focused ion-beam techniques. These developments are a reflection of the increasing significance of microstructural information in three dimensions in many fields of materials science and engineering, and four important examples are featured in this issue by Blavette et al., Marquis, Gorman et al., and Knipling et al. Indeed, it is our view that the notion of nanostructure or nanoscale microstructure is a useful concept to describe phenomena such as atomic clustering, segregation, and related fine structure involving small groups of atoms, which, together with microstructural information such as second phase composition and dispersion, grain size and structure, texture, and so on, provide a comprehensive characterization of materials. It naturally follows that high impact work will require combinations of the above techniques, and this is also a feature of the applications papers presented here. In the specific context of atom probe tomography, we have sought to feature the enabling role of ongoing hardware developments (Bunton et al.), specimen preparation (Miller et al.), as well as reconstruction and data mining approaches (Geiser et al. and Stephenson et al.). The electronic versions of most of these papers contain animations of the tomographic data associated with certain figures. To view these animations, select the "supplemental materials" for the paper at the Cambridge Journal Online website: http://journals.cambridge.org/jid_MAM.

We wish to thank all of the attendees of the Sydney workshop and to especially thank all of the contributing authors for their fine papers. Finally, we are also grateful to Professor Charles Lyman and his editorial team at Microscopy and Microanalysis for their advice, support, and encouragement for this initiative.

Guest Editors

Simon P. Ringer, The University of Sydney, Australia

David J. Larson, Imago Scientific Instruments Corp., USA

Michael P. Moody, The University of Sydney, Australia

Michael K. Miller, Oak Ridge National Laboratory, USA

Thomas F. Kelly, Imago Scientific Instruments Corp., USA 